\title{
DIELECTRIC STUDY OF DIPOLAR ASSOCIATION IN DIHALOGENOBENZENES
}

\author{
M. DutKIEWICZ \\ Institute of Physics, A. Mickiewicz University, Grunwaldzka 6, 60-780 Poznań, Poland
}

(Received June 22, 1992)

\begin{abstract}
The nonlinear dielectric effect (NDE) has been studied in o-bromochlorobenzene and $m$-bromochlorobenzene, and their solutions in benzene. On the basis of NDE results and the values of electric permittivity $\varepsilon$, density $d$, and refractive index $n$ obtained in this work, the molar polarizabilities: linear $\left\langle\mu^{2}\right\rangle$ and nonlinear $\left\langle\mu^{4}\right\rangle$ were calculated. Assuming dipolar association of the investigated dihalogenobenzenes molecules to be restricted to dimerization, the Gibbs energy $\Delta G_{\mathrm{dim}}^{0}$ of the dimerization process as a function of the Onsager field factor $f(\varepsilon)$ is determined, which allowed us to draw a conclusion on the structure of associates.
\end{abstract}

PACS numbers: $35.20 . \mathrm{My}, 61.25 . \mathrm{Em}, 82.60 . \mathrm{Hc}$

\section{Introduction}

The studies of physicochemical properties of liquids are of great importance because a majority of chemical reactions and various physicochemical processes occur in solution. The solvent effects on reaction rates and equilibria are usually described in terms of solvent polarity (for review see [1]), determined by the general solvation capability of the solvent, which in turn depends on the action of all possible intermolecular interactions taking place in a liquid. These interactions may reveal themselves in molecular association, which become more complicated if we deal with liquid mixtures. Even in the simplest case of polar/nonpolar solvent mixtures we have to take into account a dependence of molecular association which could take place in polar component, on its concentration in nonpolar solvent, which in consequence may influence the solvation ability of a dipolar solvent $[2,3]$.

One of the most sensitive methods for studies of molecular interactions in liquids is the nonlinear dielectric effect (NDE), which is manifested as a change in electric permittivity $\Delta \varepsilon=\varepsilon_{E}-\varepsilon$ of a solvent due to a strong electric field $E$. A measure of NDE is the parameter $\Delta \varepsilon / E^{2}$. The NDE studies have permitted obtaining much highly relevant information about inter- and intramolecular interactions resulting in hydrogen-bond, charge transfer and dipolar association and conformational equilibria in liquids [4-12]. 
The aim of this work was to employ the NDE method to study dipolar association in two binary mixtures of polar+nonpolar solvents, whose dipolar component molecules are isomeric, namely: $a$-bromochlorobenzene+benzene and $m$-bromochlorobenzene+benzene.

\section{Experimental}

NDE measurements were carried out with the automatically recording pulse device described in Ref. [13]. The values of $\Delta \varepsilon / E^{2}$ were determined at a frequency of ca. $2 \mathrm{MII} z$ with an accuracy of $\pm 3 \%$. The electric permittivity $\varepsilon$ was measured with an accuracy of $\pm 0.1 \%$ by the beat technique. Density $d$ was measured pyknometrically with an accuracy of $\pm 0.01 \%$. The refractive index $n$ (for the sodium $\mathrm{D}$ line) was determined with an accuracy of $\pm 0.01 \%$ with a Pulfrich refractometer. All measurements were performed at $293 \mathrm{~K}$. The concentration of dihalogenobenzene in benzene is expressed in mole fraction $f$. All solvents were purified and dried before the measurements.

\section{Results and discussion}

The experimentally obtained values of electric permittivity $\varepsilon$, density $d$, and refractive index $n$ for $o$-bromochlorobenzenc $(o-B C B)$ in benzene and for $m$-bromochlorobenzene $(m-\mathrm{BCB})$ in benzene are listed in Table.

Figure 1 shows $\Delta \varepsilon / E^{2}$ as a function of concentration $f$ of dihalogenobenzenes in benzene.

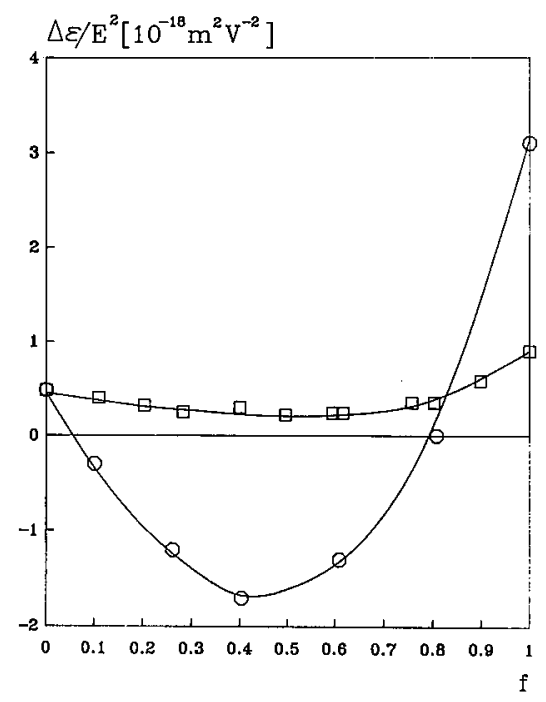

Fig. 1. $\Delta \varepsilon / E^{2}$ versus concentration $f$ of $o$-bromochlorobenzene $(0)$ and $m$-bromochlorobenzene( $\square)$ in benzene. 
TABLE

Electric permittivity $(\varepsilon)$, density $(d)$ and refractive index $(n)$ of bromochlorobenzenes in benzene.

\begin{tabular}{|c|c|c|c|c|}
\hline Solution & $\begin{array}{c}f \\
\text { [mole fraction] }\end{array}$ & $\varepsilon$ & $\begin{array}{c}d \\
{\left[\mathrm{~kg} \mathrm{~m}^{-3}\right]}\end{array}$ & $n$ \\
\hline $\begin{array}{l}\text { o-bromochloro- } \\
\text { benzene in } \\
\text { benzene }\end{array}$ & $\begin{array}{l}0.0000 \\
0.0516 \\
0.0807 \\
0.1011 \\
0.1474 \\
0.1960 \\
0.2610 \\
0.4031 \\
0.6093 \\
0.8068 \\
1.0000\end{array}$ & $\begin{array}{c}2.282 \\
2.665 \\
2.863 \\
3.015 \\
3.347 \\
3.688 \\
- \\
5.067 \\
6.463 \\
7.730 \\
9.053\end{array}$ & $\begin{array}{c}878.9 \\
- \\
- \\
979.3 \\
- \\
- \\
1124.6 \\
1243.6 \\
1389.1 \\
1537.3 \\
1658.6\end{array}$ & $\begin{array}{c}1.50124 \\
- \\
- \\
1.51152 \\
- \\
- \\
1.52647 \\
1.53893 \\
1.55672 \\
1.56941 \\
1.58312\end{array}$ \\
\hline $\begin{array}{l}m \text {-bromochloro- } \\
\text { benzene in } \\
\text { benzene }\end{array}$ & $\begin{array}{l}0.0503 \\
0.0800 \\
0.1024 \\
0.1484 \\
0.1911 \\
0.2603 \\
0.4098 \\
0.6037 \\
0.7996 \\
1.0000\end{array}$ & $\begin{array}{c}2.448 \\
2.541 \\
2.611 \\
2.758 \\
2.885 \\
3.062 \\
3.514 \\
- \\
4.565 \\
4.906\end{array}$ & $\begin{array}{c}- \\
- \\
978.2 \\
- \\
- \\
1127.9 \\
1242.1 \\
1388.6 \\
1535.3 \\
1630.0\end{array}$ & $\begin{array}{c}- \\
- \\
1.51028 \\
- \\
- \\
1.52358 \\
1.53587 \\
1.55079 \\
1.56064 \\
1.57694\end{array}$ \\
\hline
\end{tabular}

The dependence $\Delta \varepsilon / E^{2}$ vs. $\int$ for $o-B C B$ is characteristic of numerous aromatic solvents $[4,6,10]$ with negative values of NDE parameter in a wide concentration range of solution and with positive NDE for higher concentrations and pure dipolar solute. $\Delta \varepsilon / E^{2}$-values for $m$-BCB solutions are positive in the whole concentration range, although a minimum of $\Delta \varepsilon / E^{2}$ is also observed.

The different behaviour of the two isomeric compounds in the electric ficld may be explained as follows: the nonlincar dielectric effect (NDE) consists of two main phenomena: (i) the orientational Langevin effect (negative NDE) and (ii) the effect of electric field on molecular equilibria (e.g. monomer-dimer) causing a shift towards a more polar form of molecular species (positive NDE). The magnitude of contributions of two molecular effects depend on the concentration of dihalogenobenzene in benzene. In the case of $m$-BCB the (ii) effect scems to pre- 
dominate in the whole concentration range, which is observed as positive NDE, whereas for $0-\mathrm{BCB}$ the orientational effect predominates in the lower concentration range $(f<0.25)$, which is observed as negative NDE (Fig. 1). With increasing concentration of $o-\mathrm{BCB}(f>0.25)$ the effect becomes "less negative" reaching positive values of $\Delta \varepsilon / E^{2}$ for higher concentrations of $o-B C B$. Because, as mentioned above, the positive NDE depends on the state of molecular equilibria, the different behaviour of ortho- and meta-bromochlorobenzenes may result from different molecular interactions caused by a different geometry of molecules of the two isomers. The non-planarity of o-BCB molecules resulting from ortho-position of $\mathrm{Br}$ and $\mathrm{Cl}$ atoms in $\mathrm{o}-\mathrm{BCB}$ may cause an association process more complicated than in the case of $m$-BCB.

The experimental quantities obtained in this work can be expressed in terms of the two molar quantities, namely the molar dipolar polarization $\left\langle\mu^{2}\right\rangle$ expressing a mean square value of dipole moment and the nonlinear molar polarizability $\left\langle\mu^{4}\right\rangle$. With the Onsager local field model [14] these quantities can be expressed as follows:

$$
\left\langle\mu^{2}\right\rangle=\left(9 k T \varepsilon_{0} V / N\right)\left[\left(\varepsilon-n^{2}\right)\left(2 \varepsilon+n^{2}\right) / \varepsilon\left(n^{2}+2\right)^{2}\right]
$$

and

$$
\left\langle\mu^{4}\right\rangle=-\left(45 k^{3} T^{3} \varepsilon_{0} V / N\right)\left[\left(2 \varepsilon^{2}+n^{4}\right)\left(2 \varepsilon+n^{2}\right)^{2} / \varepsilon^{4}\left(n^{2}+2\right)^{4}\right]\left(\Delta \varepsilon / E^{2}\right)
$$

with $k$-Boltzmann constant, $T$ - Kelvin temperature, $\varepsilon_{0}$ - electric permittivity of vacuum, $V$ - molar volume and $N-$ Avogadro number. Formulae for the exact calculation of $\left\langle\mu^{2}\right\rangle$ and $\left\langle\mu^{4}\right\rangle$ for solutions are to be found in Ref. [15].

Figures 2 and 3 show $\left\langle\mu^{2}\right\rangle$ and $\left\langle\mu^{4}\right\rangle$ as functions of the concentration $f$ of $o$-bromochlorobenzene and $m$-bromochlorobenzene in benzene.

The change of $\left\langle\mu^{2}\right\rangle$ and $\left\langle\mu^{4}\right\rangle$ with composition gives independent information on the association in the liquid state; $\left\langle\mu^{2}\right\rangle$ vs. $f$ shows an influence of a nonpolar solvent on molecular equilibria (e.g. monomer-dimer) under field-free conditions, whercas $\left\langle\mu^{4}\right\rangle$ vs. $f$ takes into account also the electric field effect on those equilibria.

We can notice that in the lower concentration range $(f<0.25)$ the dependence of $\left\langle\mu^{2}\right\rangle$ and $\left\langle\mu^{4}\right\rangle$ on $f$ is similar for both investigated compounds showing a monotonic increase of $\left\langle\mu^{2}\right\rangle$ and $\left\langle\mu^{4}\right\rangle$ with decreasing of BCB concentrations, reaching gas values of dipole moments $\mu_{\mathrm{g}}$ at infinite dilution, which indicates that the number of monomers increases with dilution. (Gas dipole moments are: $\mu_{\mathrm{g}}(o-\mathrm{BCB})=7.3 \times 10^{-30} \mathrm{C} \mathrm{m}$, hence $\mu_{\mathrm{g}}^{2}=53.3 \times 10^{-60} \mathrm{C}^{2} \mathrm{~m}^{2}$ and $\mu_{\mathrm{g}}^{4}=$ $2840 \times 10^{-120} \mathrm{C}^{4} \mathrm{~m}^{4} ; \mu_{\mathrm{g}}(m-\mathrm{BCB})=5 \times 10^{-30} \mathrm{C} \mathrm{m}$, hence $\mu_{\mathrm{g}}^{2}=25 \times 10^{-60} \mathrm{C}^{2} \mathrm{~m}^{2}$ and $\mu_{\mathrm{g}}^{4}=625 \times 10^{-120} \mathrm{C}^{4} \mathrm{~m}^{4}$.) The decrease of $\left\langle\mu^{2}\right\rangle$ with increasing concentration (for the region below 0.25 mole fraction) of $o$-BCB as well as $m$-BCB (Fig. 2) can be explained as connected with a dimerization process taking place in a solution.

For concentrations above ca. 0.25 mole fraction, $\left\langle\mu^{2}\right\rangle$ hardly depends on concentration, for $m$ - $\mathrm{BCB}$ it monotonically decreases with increasing concentration, whereas for $o-\mathrm{BCB}$ it slightly increases. It is interesting to notice a change of the "slope" of the curve $\left\langle\mu^{4}\right\rangle$ vs. $f$ for 0 -BCB near 0.25 mole fraction (Fig. 3), which corresponds the concentration area where $\Delta \varepsilon / E^{2}$ becomes "less negative" (Fig. 1).

The obtained molar polarizabilities $\left\langle\mu^{2}\right\rangle$ and $\left\langle\mu^{4}\right\rangle$ can be interpreted assuming dipolar association of the investigated dihalogenobenzenes molecules to be 

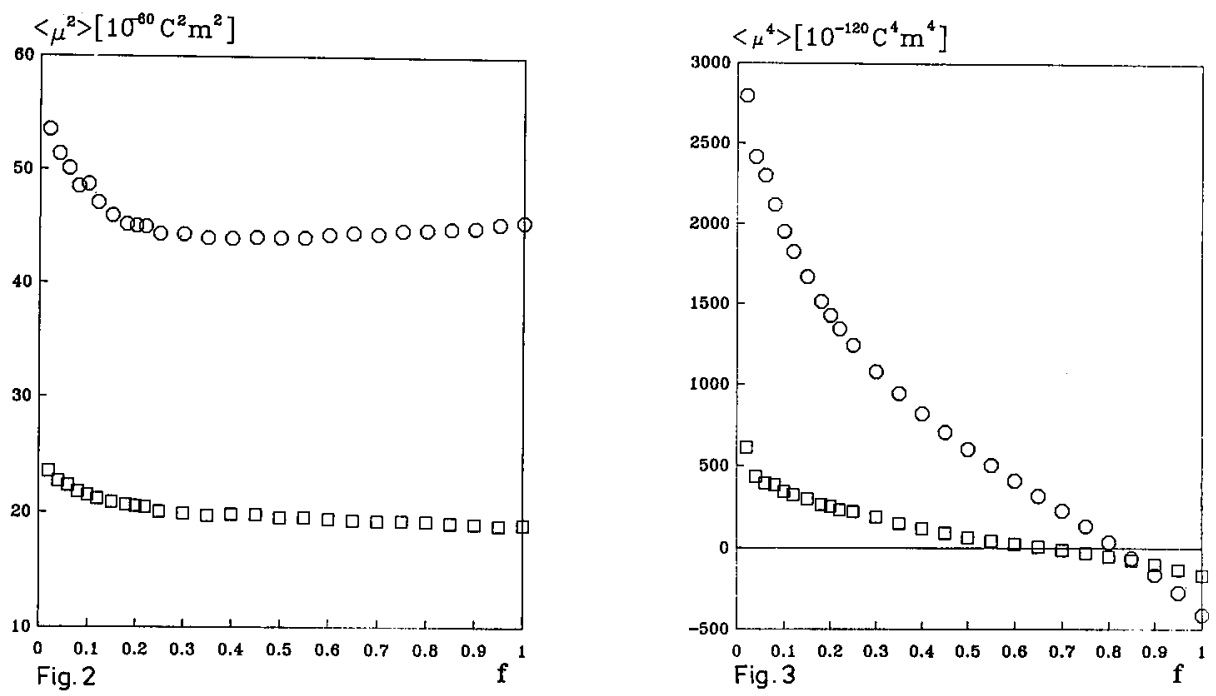

Fig. 2. The molar dipolar polarization $\left\langle\mu^{2}\right\rangle$ of $o$-bromochlorobenzene(o) and $m$-bromochlorobenzene( $\square$ ) versus concentration $f$ in benzene.

Fig. 3. The nonlinear molar polarizability $\left\langle\mu^{4}\right\rangle$ of 0 -bromochlorobenzene(o) and $m$-bromochlorobenzene( $(\square)$ versus concentration $f$ in benzene.

restricted to dimerization as the most probable first step of dipolar association. According to the general statistical theory of NDE $[8,11],\left\langle\mu^{2}\right\rangle$ and $\left\langle\mu^{4}\right\rangle$ are related to dipole moments and concentrations of associates. For dimerization process assumed in this work:

and

$$
\left\langle\mu^{2}\right\rangle=\mu_{\text {mon }}^{2}+x_{\text {dim }} \delta \mu^{2}
$$

$$
\left\langle\mu^{4}\right\rangle=\mu_{\text {mon }}^{4}+x_{\text {dim }}\left[\delta \mu^{4}-0.4\left(\delta \mu^{2}\right)^{2} / F\right]
$$

with

$$
\delta \mu^{2}=\left(\mu_{\mathrm{dim}}^{2}-2 \mu_{\mathrm{mon}}^{2}\right), \delta \mu^{4}=\left(\mu_{\mathrm{dim}}^{4}-2 \mu_{\mathrm{mon}}^{4}\right)
$$

and

$$
F=\left(1+2 x_{\operatorname{dim}}\right) /\left(1-2 x_{\operatorname{dim}}\right)
$$

where $\mu_{\text {mon }}, \mu_{\mathrm{dim}}, x_{\text {mon }}$ and $x_{\mathrm{dim}}$ are dipole moments and concentrations (in mole fractions) of monomer and dimer, respectively.

The equilibrium constant of a dimerization process

$$
K_{\text {dim }}=x_{\text {dim }} / x_{\text {mon }}^{2},
$$

can then be calculated. $K_{\text {dim }}$ is directly related to the change in the Gibbs energy:

$$
\Delta G_{\text {dim }}^{0}=-R T \ln K_{\text {dim }} \text {. }
$$

Figures 4 and 5 show $\Delta G_{\mathrm{dim}}^{0}$ (calculated according to Refs. $[8,11]$ and with Eqs. (3) to (6) of this work) for the investigated dihalogenobenzenes plotted as a function of the Onsager reaction field parameter: $f(\varepsilon)=(\varepsilon-1) /(2 \varepsilon+1)$. 

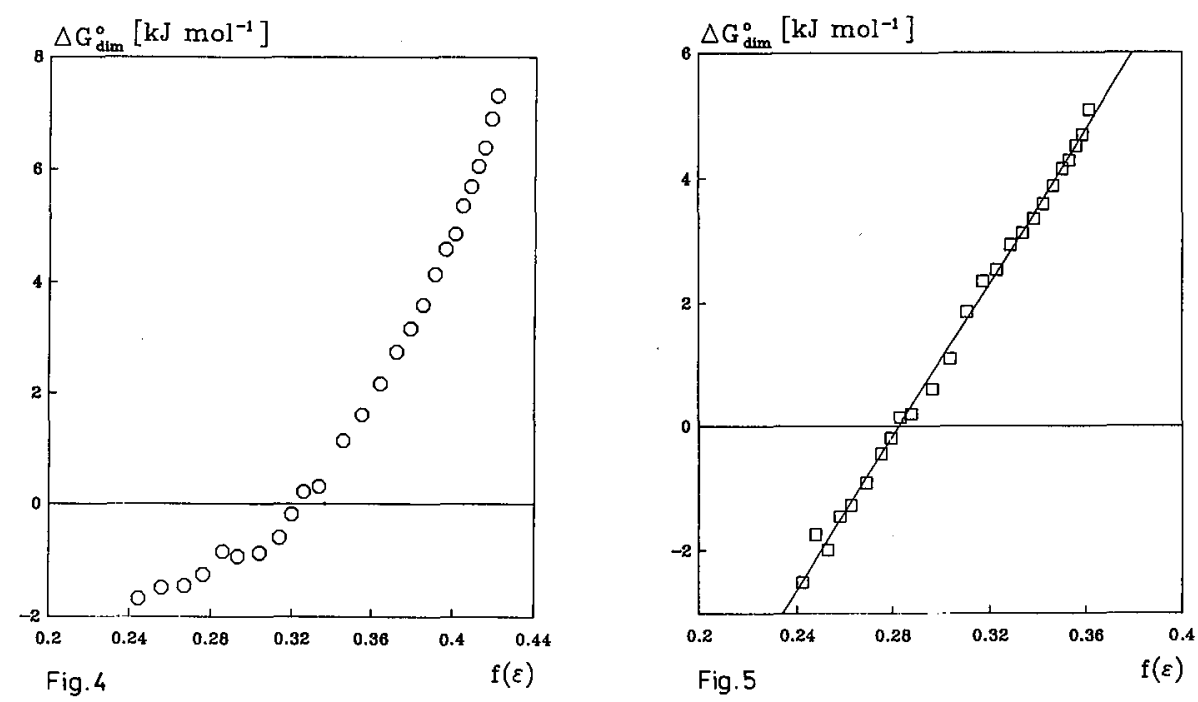

Fig. 4. The Gibbs energy $\Delta G_{\mathrm{dim}}^{0}$ of the dimerization process in o-bromochlorobenzene versus $f(\varepsilon)$ parameter.

Fig. 5. The Gibbs energy $\Delta G_{\mathrm{dim}}^{0}$ of the dimerization process in $m$-bromochlorobenzene versus $f(\varepsilon)$ parameter.

For both dihalogenobenzenes $\Delta G_{\mathrm{dim}}^{0}$ increases with increasing $f(\varepsilon)$, for 0 -BCB the function is nonlinear, whereas for $m$-BCB it is linear. The increase of $\Delta G_{\mathrm{dim}}^{0}$ with $f(\varepsilon)$ can be explained taking into account the electric field effect on molecular species (monomers and dimers) whose existence is assumed in solution. As the electric field favours more polar species, so the higher $f(\varepsilon)$, the higher $\Delta G_{\mathrm{dim}}^{0}$ and the higher is monomer concentration (Eqs. (5) and (6)). It means that in this case dimer is a less polar form so it should have a configuration of antiparallel dipoles with the net dipole moment close to zero. Ilowever, the nonlinearity of $\Delta G_{\mathrm{dim}}^{0}$ vs. $f(\varepsilon)$ (Fig. 4) seems to indicate that the assumption of a simple monomer-dimer equilibrium may be a simplification in the case of $a-B C B$.

\section{Conclusions}

On the basis of experimentally obtained results, molecular association in ortho- and meta-bromochlorobenzenes was interpreted in terms of the monomer-dimer equlilibrium. This approach has permitted to obtain a satisfactory description of the association process taking place in the studied liquids, although we have to keep in mind that in such weakly dipolar liquids we deal with very labile molecular species. Taking into account this fact we are aware that it would be better if we could consider not the equilibrium between monomers and dimers but the equlibrium between associated and non-associated part of the dipolar solvent instead, but at the moment, we are not able to follow such a way to interpret the association in aprotic dipolar liquids. The different dielectric behaviour of ortho- and 
meta-bromochlorobenzenes indicates a significant role of geometry of the molecule in the association process.

\section{References}

[1] C. Reichardt, Solvents and Solvent Effects in Organic Chemistry, 2nd Ed., Verlag Chemie, Weinheim 1988.

[2] V.P. Senthilnathan, M. Kanakavel, Surjit Singh, Spectrochim. Acta A 30, 285 (1974).

[3] E. Dutkiewicz, A. Jakubowska, M. Dutkiewicz, Spectrochim. Acta A, in press.

[4] A. Pickara, Proc. R. Soc. Lond. A 172, 360 (1939); Acla Phys. Pol. 10, 37 and 107 (1950).

[5] S. Kielich, in: Dielectric and Relaled Molecular Processes, Vol. 1, Ed. M. Davies, Chem. Soc., London 1972, p. 192.

[6] A. Chelkowski, Dielectrics Physics, Elscvier, Amsterdam 1980.

[7] G. Parry Jones, in: Dielectric and Related Molecular Processes, Vol. 2, Ed. M. Davies, Chem. Soc., London 1975, p. 198 and cit. therein.

[8] J. Malecki, J. Chem. Soc. Faraday Trans. II 72, 104 (1976).

[9] J. Nowak, J. Malecki, J.M. Thiebaut, J.L. Rivail, J. Chem. Soc. Faraday Trans. II 76, 197 (1980).

[10] M. Dutkiewicz, J. Chem. Soc. Faraday Trans. II 77, 1301 (1981); J. Mol. Liq. 28, 147 (1984); Chem. Phys. Lett. 121, 73 (1985).

[11] J. Malecki, Electrochim. Acla 33, 1235 (1988).

[12] J. Malecki, J. Nowak, J. Mol. Strucl. 248, 373 (1991).

[13] J. Malecki, J. Chem. Soc. Faraday Trans. II 72, 1214 (1976).

[14] L. Onsager, J. Am. Chem. Soc. 58, 1486 (1936).

[15] M. Dutkicwicz, Chem. Phys. Lett. 112, 177 (1984). 